\title{
Variable $m$-CAP for Bandlimited Visible Light Communications
}

\author{
Petr Chvojka ${ }^{1}$, Stanislav Zvanovec ${ }^{1}$, Khald Werfli ${ }^{2}$, Paul Anthony Haigh ${ }^{3}$ and Zabih Ghassemlooy ${ }^{2}$ \\ ${ }^{1}$ Department of Electromagnetic Field, Faculty of Electrical Engineering, Czech Technical University in Prague, Czech Republic \\ ${ }^{2}$ Optical Communications Research Group, NCRLab, Faculty of Engineering and Environment, Northumbria University, \\ Newcastle upon Tyne, NE1 8ST, UK \\ ${ }^{3}$ Communications and Information Systems, University College London, Gower Street, London, WC1E 6BT, UK \\ Corresponding author: chvojpe8@fel.cvut.cz
}

\begin{abstract}
The multi-band carrier-less amplitude and phase ( $m$-CAP) modulation technique has been demonstrated as a significant candidate for use in visible light communication (VLC) systems, as spectral efficiency becomes ever more important. When employing $m$-CAP modulation, the signal bandwidth is typically split into $m$ equally distributed subcarriers regardless of the $-3 \mathrm{~dB}$ bandwidth. Thus, in this paper we investigate the impact of splitting the signal bandwidth into unequally spaced subcarriers on the $m$-CAP VLC system performance. We demonstrate that by allocating different bandwidths for individual subcarriers, we can achieve up to $36 \%$ improvement in data rate for 6-CAP (i.e. 6 subcarriers). We show that the higher the order of the variable $m$ CAP, the more significant improvement in data rate becomes even for a highly bandlimited VLC system when compared to the conventional $m$-CAP scheme.
\end{abstract}

Keywords-carrier-less amplitude and phase modulation, visible light communications

\section{INTRODUCTION}

Mobile data traffic is expected to increase eight times by 2020 in comparison to 2015 , demonstrating exponential growth [1]. This is due to the increasing number of smart devices (expected to cover nearly 50\% of the global market in 2020). Demand for multimedia content broadcasting will boost requirements for communication infrastructures with high spectral efficiency and transmission speeds. These challenges can be addressed by adopting optical wireless communications using light-emitting diodes (LEDs) as the transmitters, which is known as visible light communications (VLC). VLC offers multiple functionalities including illumination, high-speed data communication over short range distances of several meters and indoor positioning $[2,3,4]$.

VLC utilizes white LEDs, which are highly energy efficient with a fast switching capability. However, their modulation bandwidth is limited to just a few $\mathrm{MHz}$ and reduces the maximum achievable bit rates in many applications. Therefore to address this shortcoming and fulfil the demands for the increasing data traffic, it is essential to emphasize the necessity of methods for improving the following areas within VLC systems: (i) multi-level modulations [5]; (ii) filtering and equalization [6] and (iii) spectrally efficient modulation formats such as optical orthogonal frequency division multiplexing (OFDM) and multiband carrier-less amplitude and phase
(m-CAP) modulation. The OFDM technique can be optimized based on the channel conditions using various bit- and powerloading algorithms to improve the communication performance [7]. However, OFDM based VLC systems are sensitive to the nonlinear electro-optical characteristics of LEDs, due to their high peak-to-average power ratio (PAPR), which thus leads to system performance degradation [8].

Besides OFDM, the $m$-CAP modulation technique has recently been the focus of extensive investigation [9-14]. The multiband form of CAP, which splits the total signal bandwidth into $m$ equally spaced subcarriers, was developed for optical fibre communication systems, showing enhanced performance over a traditional 1-CAP in terms of bandwidth efficiency, dispersion, reduced sampling rate and slightly increased data rate [10]. The first $m$-CAP based VLC system was experimentally investigated in [11] showing the spectral efficiency of $4.85 \mathrm{~b} / \mathrm{s} / \mathrm{Hz}$ for $10-\mathrm{CAP}$, which was a new record at the time of publication. Such a promising result has led to further investigation of $m$-CAP performance. For instance, numerical simulations of $m$-CAP in a highly bandlimited VLC link were reported in [12] showing $40 \%$ improvement in data rate over traditional 1-CAP technique when 10-CAP is employed. The work presented in [13] focused on the analysis of pulse shaping filters, which are important for both overall system performance and complexity that is crucial for the development of VLC systems in real environments. Note that $2 m$ filters must be used in both the transmitter and the receiver side, giving an overall $4 m$ filter requirement per deployment. Two key filter parameters such as the roll-off factor $\alpha$ and the filter length were under investigation showing that increasing both parameters significantly enhances the bit error rate (BER) performance, especially for higher order $m$-CAP systems. To decrease the system complexity by reducing the number of pulse shaping filters, a new concept of $m$-CAP was proposed in [14]. The desirable signal bandwidth was split such that the $1^{\text {st }}$ subcarrier occupied the LED modulation bandwidth, while the remaining $m-1$ subcarriers were equally distributed within the rest of the signal bandwidth (i.e. outside of the $-3 \mathrm{~dB}$ modulation bandwidth, where the frequencies are attenuated by $20 \mathrm{~dB} /$ decade). This resulted in a reduction of the system complexity by up to $80 \%$ for a 10 -CAP system, at the cost of a slight power penalty of $1.5 \mathrm{~dB}$, thus offering a promising solution. 


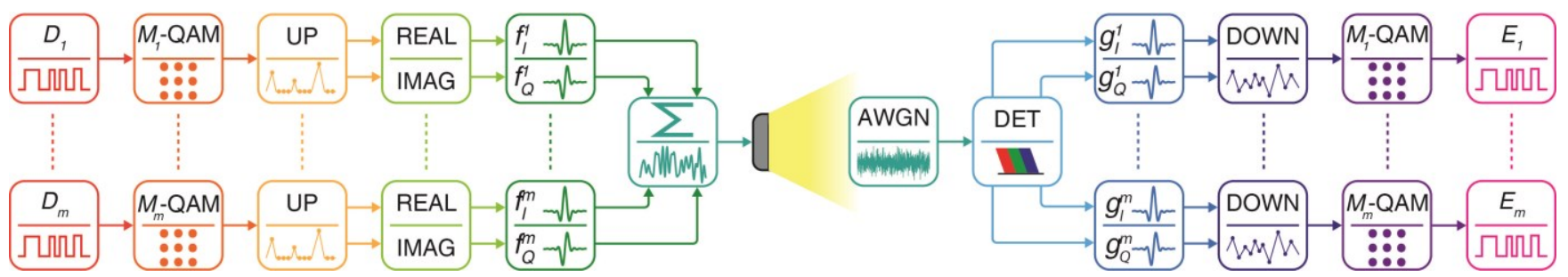

Fig. 1. The schematic block diagram of the tested system. The generated data streams $D_{m}$ are mapped into $M$-QAM constellation symbols, upsamlped ('UP'), split into it's real and imaginary parts and passed through the pulse shaping fillters. After passing the ideal low-pass filter channel (LED) with AWGN, the signal is captured by a detector ('DET') and the matched filters are utilized, down-sampling ('DOWN') and de-mapping follow and the received data is estimated (' $E_{m}$ ').

In this paper, we extend the work reported in [14] as we theoretically investigate the $m$-CAP system performance in terms of achievable bit rates by variably splitting the available signal bandwidth. We divide the signal bandwidth into $m$ subcarriers, so that $x$ subcarriers are set within the region of the LED modulation bandwidth and $y=m-x$ subcarriers are divided into the remaining part of the signal bandwidth as follows: 2-CAP $(x / y=1 / 1)$, 4-CAP $(x / y=1 / 3,2 / 2,3 / 1)$ and 6-CAP $(x / y=1 / 5,2 / 4,3 / 3,4 / 2,5 / 1)$. We show a significant improvement in the transmission speed up to $30 \%$ for the variable 6-CAP. In the best case scenario, the improvement in data rate is about $36 \%$ higher than the conventional 6-CAP. Moreover, we find that the higher the order of $m$, an LED with a lower cut-off frequency can be utilized to improve the system performance.

The paper is organized as follows. Section II describes the principle of $m$-CAP modulation and tested system model. Results are presented and discussed in Section III and finally, conclusions are given in Section IV.

\section{COMmunication System ModeL}

\section{A. m-CAP Principle}

The performance of the $m$-CAP system is investigated utilizing the test setup as illustrated in the block diagram in Fig. 1. The principle of the system will be described briefly, for a detailed description of the $m$-CAP functionality, see previous reports in the literature [9, 13]. Independent data streams $D$ are generated for each subcarrier and consequently mapped into $M$-quadrature amplitude modulation (QAM) symbols, where $M$ is the order of QAM. After up-sampling by the means of zero padding, the data is split into real and imaginary components before being passed through specifically designed real (in-phase) and imaginary (quadrature) pulse shaping transmit filters, whose impulse responses form a Hilbert pair (i.e. orthogonal in the time domain). In our system we utilize square root raised cosine (SRRC) filters with a fixed roll-off factor $\alpha$ that determines the excess bandwidth of the transmitted signal. The output signal modulating an LED intensity is given as $[12,13]$

$$
s(t)=\sqrt{2} \sum_{n=1}^{m}\left(s_{I}^{n}(t) \otimes f_{I}^{n}(t)-s_{Q}^{n}(t) \otimes f_{Q}^{n}(t)\right),
$$

where $s_{I}$ and $s_{Q}$ are the real and imaginary QAM symbols for the $n^{\text {th }}$ subcarrier and $f_{I}$ and $f_{Q}$ are the in-phase and quadrature transmit filter impulse responses, respectively, and $\otimes$ denotes time-domain convolution.

The transmitted signal is passed through an ideal $1^{\text {st }}$ order low-pass filter (LPF) channel which represents an LED with $-3 \mathrm{~dB}$ cut-off frequency $f_{c}$. The filter is modelled in the discrete time domain for compatibility with the generated signal. After passing the additive white Gaussian noise (AWGN) channel, the signal is demodulated using matched filters $g_{l}^{n}$ and $g_{Q}{ }^{n}$ at the receiver. The receiving filters are time-reversed matched versions of the transmit filters recalling that the total number of the filters used within the system is $4 m$. Finally, the signal is down-sampled and de-mapped into individual $M$-QAM symbols to recover the estimated version of input data.

\section{B. Test Setup}

Since the proposed $m$-CAP system is based on splitting of the available signal bandwidth $B_{s}$ into $m$ subcarriers, the individual subcarriers are typically distributed equally within the $B_{s}$ as illustrated in Fig. 2(a) [9, 10]. However, adopting nonuniform subcarrier spacing can result in additional system performance improvement in a bandlimited environment, which is investigated in the rest of the paper. To demonstrate this, we divided $B_{s}$ into $m$ subcarriers with $x$ subcarriers positioned within the passband region of an LED and $y=m-x$ subcarriers are divided into the remaining part of the signal bandwidth (i.e. outside the $-3 \mathrm{~dB}$ bandwidth). Thus, the transmit filters' carrier frequencies with $x / y$ distribution are given as:

$$
f_{s c}^{i}=\frac{1}{x}\left(i-\frac{1}{2}\right) \cdot B_{\text {tot }} \cdot f_{c} \quad \text { for } \quad i=1, \ldots, x
$$

for the first $x$ subcarriers and

$$
\begin{gathered}
f_{s c}^{i}=B_{\text {tot }}\left(\frac{\left(i-x-\frac{1}{2}\right) \cdot\left(1-f_{c}\right)}{y}+f_{c}\right) \\
\text { for } i=x+1, \ldots, m
\end{gathered}
$$

for the remaining $y$ subcarriers. $B_{t o t}$ is the total signal bandwidth, which is given by:

$$
B_{t o t}=B_{s} \cdot(1+\alpha)
$$

where $B_{s}$ is set in our system model equal to $1 \mathrm{MHz}$. 

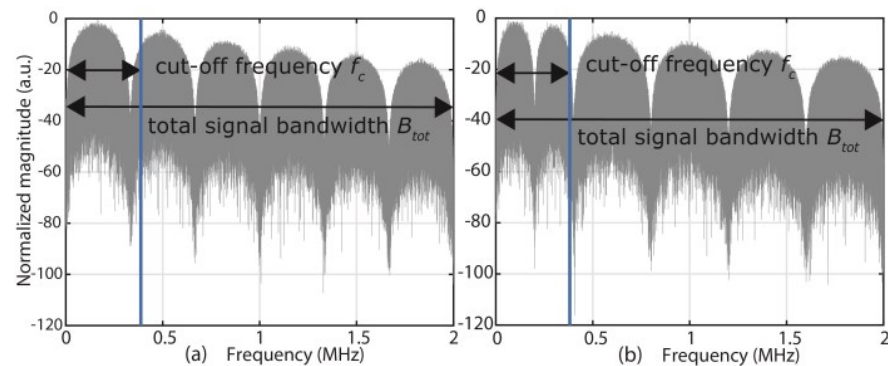

Fig. 2. The frequency spectrum of the transmitted signal for (a) $m$-CAP with equally spaced subcarriers and (b) $m_{2 / 4}$ CAP with $x=2$ and $y=4$ subcarriers in the passband region and outside the $3 \mathrm{~dB}$ bandwidth, respectively.

This new version of $m$-CAP is denoted as $m_{x / y}$-CAP and the bandwidth is divided as mentioned above, recalling: $2 x / y$-CAP $(x / y=1 / 1), 4_{x / y}$-CAP $(x / y=1 / 3,2 / 2,3 / 1)$ and $6_{x / y}$ CAP $(x / y=1 / 5$, $2 / 4,3 / 3,4 / 2,5 / 1)$. For instance, for parameters $\alpha=1, m=6$, $x=2, y=4$ and $f_{c}=0.2 \cdot B_{\text {tot }}$, the resultant $m_{2 / 4}$-CAP frequency spectrum is illustrated in Fig. 2(b) showing 2 subcarriers within an LED modulation bandwidth given by $f_{c}$ and 4 subcarriers distributed in the roll-off region of the frequency response.

The parameters used in the numerical simulations are set as follows: number of subcarriers $m=\{2,4,6\}, f_{c}=\{0.1,0.2$, $0.3,0.4,0.5\}$ to ensure the sufficient band limitation, $\alpha=1$ as in [12], since $\alpha$ does not represent a critical parameter under investigation in this work, $x=\{1, \ldots, m-1\}$ and $y=\{1, \ldots, m-1\}$ to test all possible subcarrier distributions. The performance of the system is analysed in the terms of the predicted bit rates using a bit-loading technique based on the measured error vector magnitude (EVM) [15]. Thus, the generated bit streams are mapped into binary phase shift keying (BPSK) symbols. After passing the LPF channel, the signal is demodulated and EVM measurement is performed to calculate the received signal to noise ratio (SNR) for each subcarrier as in line with [12]. The bit allocation for each subcarrier is performed using estimated SNR values according to the given BER target of $10^{-4}$, which is below the forward error correction (FEC) limit with an overhead of $7 \%\left(3.8 \times 10^{-3}\right)$. The theoretical SNR thresholds for loading a maximum number of bits/symbol/subcarrier can be found in [16].

\section{RESULTS}

This section presents the results of the numerical simulations using parameters outlined in Section II. The BPSK pilot signal is transmitted over the LPF channel for a range of input energy/bit $\left(E_{b} / N_{0}\right)$ values of $5-25 \mathrm{~dB}$. The appropriate number of bits/symbol is loaded into individual subcarriers (denoted as $s$ ) and theoretical total bit rates are calculated for every possible subcarrier distribution $x / y$ based on the measured SNR requirements. The achieved bit rates of variable $m$-CAP system are consequently subtracted from the traditional $m$-CAP for each $E_{b} / N_{0}$. The resultant values are obtained by calculating the average variation of the subtracted data from the bit rates of conventional $m$-CAP system over a range of $E_{b} / N_{0}$. In Fig. 3 , the average variations of the $21 / 1-\mathrm{CAP}$ across the range of $E_{b} / N_{0}$ compared to a conventional 2-CAP system are illustrated. Clearly, in a bandlimited channel, the splitting of the available bandwidth does not offer a performance enhancement in comparison to the conventional 2-CAP scheme. This is because

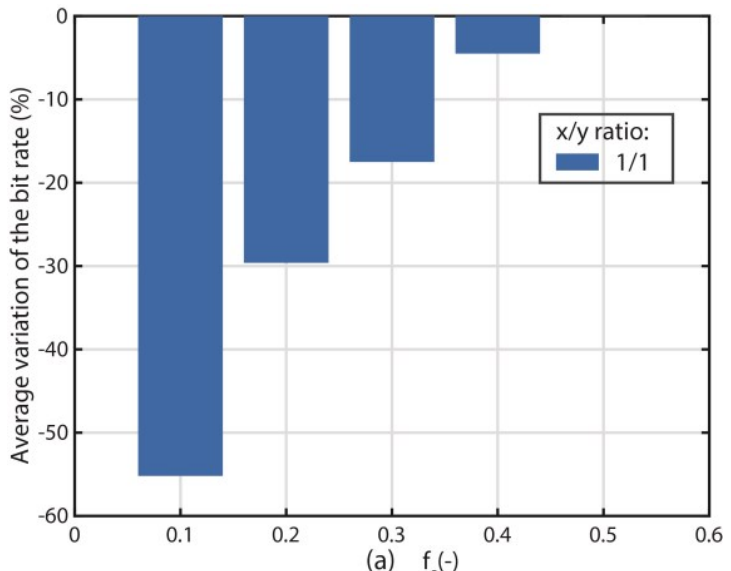

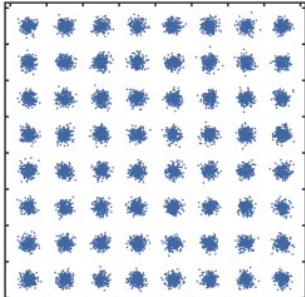

(b)

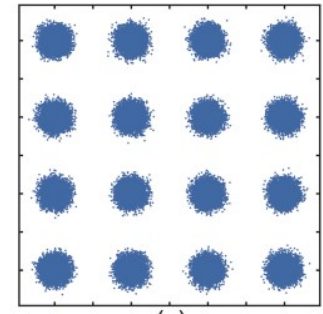

(c)
Fig. 3. (a) The average variation of the achieved total bit rate for $2_{1 / 1}$-CAP when compared to 2-CAP over the range $E_{b} / N_{0}$. Note that for $f_{c}=0.5$ the variation is zero since the available signal bandwidth is split as in the conventional 2-CAP system. The received constellation diagrams of $2_{1 / 1}$-CAP for subcarriers (b) $s=1$ and (c) $s=2$ for $f_{c}=0.3$ at $E_{b} / N_{0}=20 \mathrm{~dB}$.

for $f_{c}<0.5$ the $2^{\text {nd }}$ subcarrier has a greater bandwidth requirement and therefore is more prone to the high-frequency attenuation outside the $-3 \mathrm{~dB}$ bandwidth. Although the $1^{\text {st }}$ subcarrier is within the LED bandwidth, thus suffering a maximum attenuation of $-3 \mathrm{~dB}$, the subcarrier bandwidth is too low for high data rate transmission. However, for $f_{c}>0.5$ we observe improvement in the data rate over the traditional 2-CAP, as can be expected following the same approach. The constellation diagrams of $2{ }_{1 / 1}$-CAP for subcarriers $s=1$ (i.e., 64-QAM) and 2 (i.e., 16-QAM) for $f_{c}=0.3$ at $E_{b} / N_{0}=20 \mathrm{~dB}$ observed at the receiver are illustrated in Figs. 3(b) and 3(c), respectively.

The comparison of $4_{x / y}$-CAP and conventional 4-CAP is illustrated in Fig. 4 in terms of the average bit rate variation as a function of $f_{c}$ for $x / y=1 / 3,2 / 2$ and $3 / 1$. The best performing $4_{1 / 3}$-CAP system shows the average improvements of $10.2 \%$, $16.1 \%$ and $20.8 \%$ for $f_{c}=0.3,0.4$ and 0.5 , respectively, compared to a traditional 4-CAP communication system. This is due to assigning the entire LED $-3 \mathrm{~dB}$ bandwidth to the $1^{\text {st }}$ subcarrier. For $f_{c}=0.1$, the conventional 4-CAP outperforms the variable $m_{x / y}$-CAP system significantly by about $29.4 \%$, $36.5 \%$ and $45.5 \%$ for $x / y$ of $1 / 3,2 / 2$ and $3 / 1$, respectively, regardless of the subcarrier distributions. This is due to the higher bandwidth requirements of the subcarriers outside the $-3 \mathrm{~dB}$ bandwidth experiencing high attenuation. The comparison of the predicted bit rates between the conventional 4-CAP and $4_{1 / 3}$-CAP is shown in Fig. 4(b) for $f_{c}=0.5$. The highest improvement in the bit rate is $0.5 \mathrm{Mb} / \mathrm{s}(25 \%)$ at $E_{b} / N_{0}=18 \mathrm{~dB}$. Higher performance enhancement can be expected with 

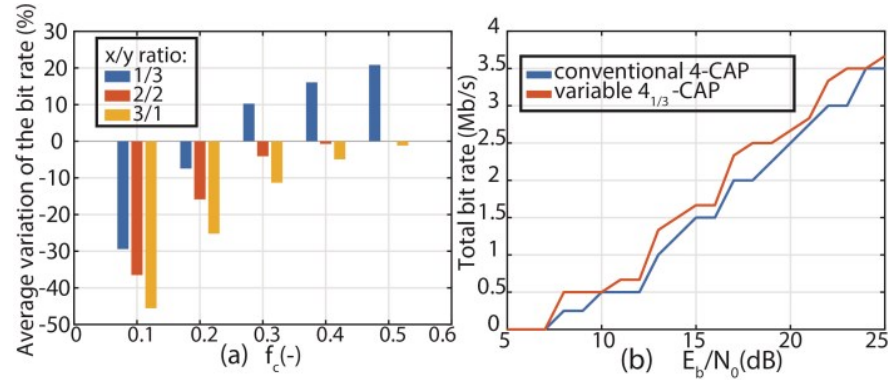

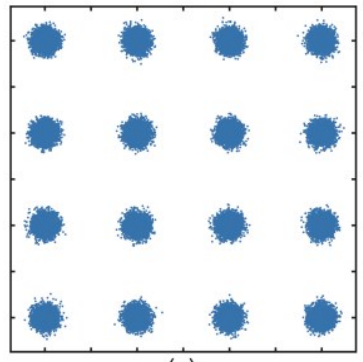

(c)

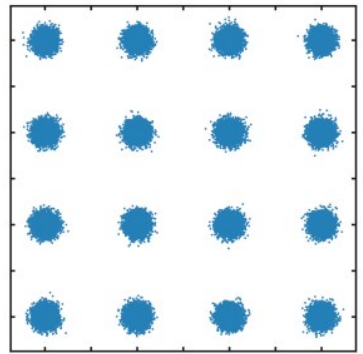

(e)

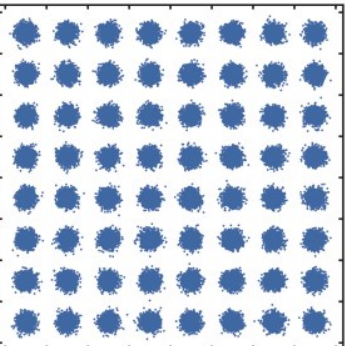

(d)

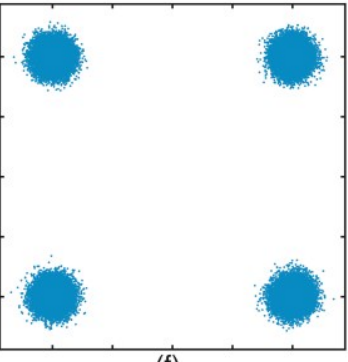

(f)
Fig. 4. (a) The average variation of the achieved total bit rate for $4_{1 / 3}$-CAP (blue), $4_{2 / 2}$-CAP (red) and $4_{3 / 1}$-CAP (yellow) when compared to the 4-CAP over the range $E_{b} / N_{0}$. Note that for $f_{c}=0.54_{2 / 2}$-CAP has the same subcarriers distribution as conventional 4-CAP. (b) The total bit rates for $4_{1 / 3^{-}}$ CAP (red) and conventional 4-CAP (blue) and the received constellation diagrams of $4_{1 / 3}$-CAP for subcarriers (c) $s=1$, (d) $s=2$, (e) $s=3$ and (f) $s=4$ for $f_{c}=0.3$ at $E_{b} / N_{0}=20 \mathrm{~dB}$.

increasing $f_{c}$. Also shown in Fig. 4 is the received constellation diagrams of $M$-QAM $4_{1 / 3}$-CAP for subcarriers $s=1,2,3$ and 4 for $f_{c}=0.3$ at $E_{b} / N_{0}=20 \mathrm{~dB}$ in Figs. 4 (c)-(f), respectively, based on the EVM measurements, showing the improvement in the performance.

The performance of $6_{x / y}-C A P$ system is illustrated in Fig. 5(a) for all possible subcarriers distributions. As expected $6_{x / y}$-CAP modulation is outperformed by conventional 6-CAP for a very low value of $f_{c}=0.1$. However, $6_{1 / 5}$-CAP shows enhanced performance even for $f_{c}=0.2$ and a significant improvement in the average bit rate for higher values of $f_{c}$. In the best case scenario, the average data rate enhancement is $\sim 30 \%$ for $f_{c}=0.4$ as illustrated in Fig. 5(b). The best data rate achieved with the proposed system is $36 \%(\sim 0.54 \mathrm{Mb} / \mathrm{s})$ higher than the traditional 6-CAP at $E_{b} / N_{0}=17 \mathrm{~dB}$. For $f_{c}=0.4$ all the possible subcarrier distributions offer superior performance in comparison to the conventional 6-CAP system because of reduced bandwidth requirements for subcarriers residing beyond the $-3 \mathrm{~dB}$ bandwidth. To further illustrate the variable system performance, the received constellation diagrams are shown in Figs. 5 (c)-(h) for $s=1,2,3,4,5$ and 6, respectively for $f_{c}=0.3$ at $E_{b} / N_{0}=20 \mathrm{~dB}$.
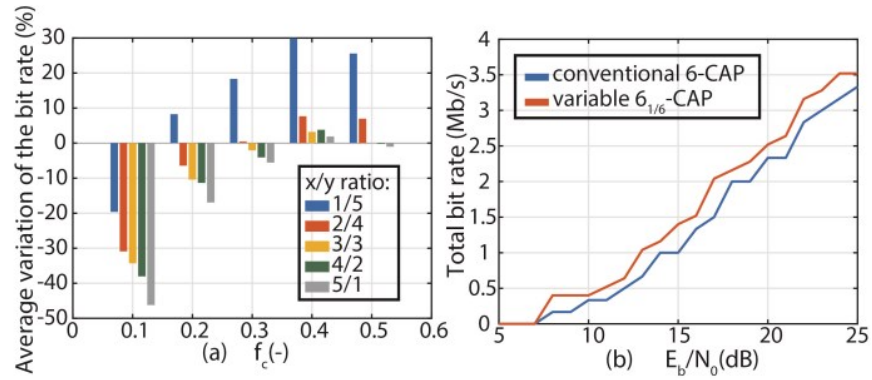

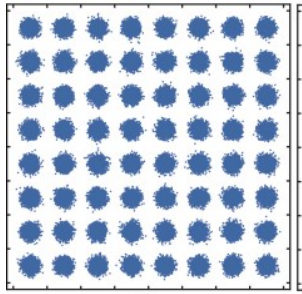

(c)

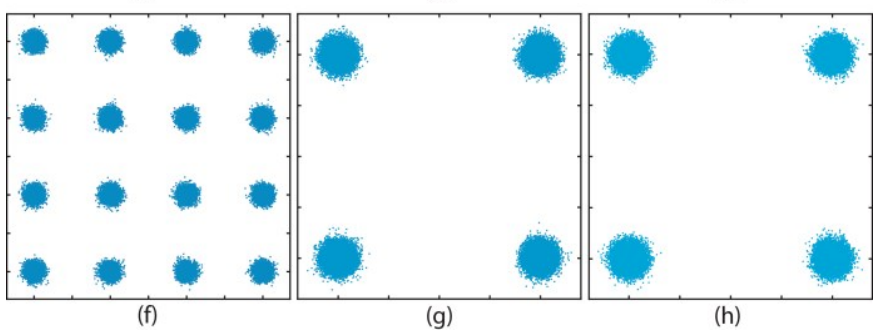

Fig. 5. (a) The average variation of the achieved total bit rate for $6_{1 / 5}$-CAP (blue), $6_{2 / 4}$-CAP (red), $6_{3 / 3}$-CAP (yellow), $6_{4 / 2}$-CAP (green) and $6_{5 / 1}$-CAP (grey) when compared to the conventional 6-CAP over the entire range $E_{b} / N_{0}$. Note that $6_{3 / 3}$-CAP has the same subcarriers distribution as conventional 6-CAP and hence the difference is zero. (b) The total bit rates for $6_{1 / 5}$-CAP (red) and conventional 6-CAP (blue). The received constellation diagrams of $6_{1 / 5}$-CAP for subcarriers (c) $s=1$, (d) $s=2$, (e) $s=3$, (f) $s=4,(\mathrm{~g})=5$ and (h) $=6$ for $f_{c}=0.3$ at $E_{b} / N_{0}=20 \mathrm{~dB}$.

The results also show that increasing the number of subcarriers (i.e., $m$ ) results in higher bit rates in the proposed variable system compared to the conventional $m$-CAP scheme. We also demonstrated that in VLC, with its' inherent bandwidth limitations, employing a higher order of $m$-CAP system leads to increased data rates, which is desirable in many applications. Moreover, the best performing subcarrier distribution $1 / y$ offers much reduced system complexity as outlined in [14].

\section{CONCLUSIONS}

The possibility of variable subcarriers distribution strategy within the available signal bandwidth was investigated for the first time for $m$-CAP VLC system, showing considerable improvement in the achievable bit rates. The variable system offered the average and the maximum improvement of $\sim 30 \%$ and $36 \%$ in bit rates for 6 subcarriers in comparison to conventional 6-CAP.

In our future work we aim to investigate further on this new variable $m$-CAP system under various roll-off factor parameters and filter lengths before experimental validation. 


\section{ACKNOWLEDGMENT}

This work was supported by GACR 17-17538S and the UK EPSRC grant EP/P006280/1: Multifunctional Polymer LightEmitting Diodes with Visible Light Communications (MARVEL) and the presentation was supported by CTU grant SGS17/182/OHK3/3T/13.

\section{REFERENCES}

[1] -, "Cisco Visual Networking Index: Global Mobile Data Traffic Forecast Update, 2014-2019, White paper," ed, 2013.

[2] H. Chun et al., "LED Based Wavelength Division Multiplexed $10 \mathrm{~Gb} / \mathrm{s}$ Visible Light Communications," in Journal of Lightwave Technology, vol. 34, no. 13, pp. 3047-3052, July1, 12016.

[3] J. Armstrong, Y. A. Sekercioglu and A. Neild, "Visible light positioning: a roadmap for international standardization," in IEEE Communications Magazine, vol. 51, no. 12, pp. 68-73, December 2013.

[4] Z. Ghassemlooy, L. N. Alves, S. Zvanovec and M. Ali Khalighi, Visible Light Communications: Theory and Applications, CRC Press, 2017 (to be published).

[5] S. H. Lee, K. I. Ahn and J. K. Kwon, "Multilevel Transmission in Dimmable Visible Light Communication Systems," in Journal of Lightwave Technology, vol. 31, no. 20, pp. 3267-3276, Oct.15, 2013.

[6] Victor S. C. Teichmann, Andre N. Barreto, Tien-Thang Pham, Roberto Rodes, Idelfonso T. Monroy, and Darli A. A. Mello, "SC-FDE for MMF short reach optical interconnects using directly modulated $850 \mathrm{~nm}$ VCSELs," Opt. Express 20, 25369-25377 (2012).

[7] D. Bykhovsky and S. Arnon, "An experimental comparison of different bit-and-power-allocation algorithms for DCO-OFDM," Journal of Lightwave Technology, vol. 32, no. 8, pp. 1559-1564, 2014.

[8] R. Mesleh, H. Elgala and H. Haas, "LED nonlinearity mitigation techniques in optical wireless OFDM communication systems," in
IEEE/OSA Journal of Optical Communications and Networking, vol. 4, no. 11 , pp. $865-875$, Nov. 2012

[9] P. A. Haigh, L. Son Thai, S. Zvanovec, Z. Ghassemlooy, L. Pengfei, X. Tongyang, et al., "Multi-band carrier-less amplitude and phase modulation for bandlimited visible light communications systems," Wireless Communications, IEEE, vol. 22, pp. 46-53, 2015.

[10] M. I. Olmedo, Z. Tianjian, J. B. Jensen, Z. Qiwen, X. Xiaogeng, S. Popov, et al., "Multiband Carrierless Amplitude Phase Modulation for High Capacity Optical Data Links," Lightwave Technology, Journal of, vol. 32, pp. 798-804, 2014.

[11] P. A. Haigh, A. Burton, K. Werfli, H. L. Minh, E. Bentley, P. Chvojka, et al., "A Multi-CAP Visible Light Communications System with 4.85 b/s/Hz Spectral Efficiency," Selected Areas in Communications, IEEE Journal on, vol. PP, pp. 1-1, 2015

[12] P. A. Haigh et al., "Multi-band carrier-less amplitude and phase modulation for highly bandlimited visible light communications Invited paper," Wireless Communications \& Signal Processing (WCSP), 2015 International Conference on, Nanjing, 2015, pp. 1-5.

[13] P. Chvojka, P. A. Haigh, S. Zvanovec, P. Pesek and Z. Ghassemlooy, "Evaluation of Multi-band Carrier-less Amplitude and Phase Modulation Performance for VLC under Various Pulse Shaping Filter Parameters," in Proceedings of the 13th International Joint Conference on e-Business and Telecommunications, pp. 25-31.

[14] K. Werfli, P. A. Haigh, Z. Ghassemlooy, N. B. Hassan and S. Zvanovec, "A new concept of multi-band carrier-less amplitude and phase modulation for bandlimited visible light communications," 2016 10th International Symposium on Communication Systems, Networks and Digital Signal Processing (CSNDSP), Prague, 2016, pp. 1-5.

[15] R. Schmogrow et al., "Error Vector Magnitude as a Performance Measure for Advanced Modulation Formats," in IEEE Photonics Technology Letters, vol. 24, no. 1, pp. 61-63, Jan.1, 2012.

[16] J. G. Proakis, Digital Communications. New York: McGraw - Hill, 2004. 